\title{
La remise au jour du Buddha de Vat Kompong Luong
}

Bertrand Porte

\section{Citer ce document / Cite this document :}

Porte Bertrand. La remise au jour du Buddha de Vat Kompong Luong. In: Arts asiatiques, tome 57, 2002. pp. 219-222;

doi : https://doi.org/10.3406/arasi.2002.1491

https://www.persee.fr/doc/arasi_0004-3958_2002_num_57_1_1491

Fichier pdf généré le 21/04/2018 
Un Buddha assis à l'indienne, haut de 1,2 m, provient de la pagode de Vat Kompong Luong, mais il a subi des additions et des dorures qu'on ne peut enlever actuellement pour des raisons religieuses et qui rendent son examen très imparfait».

(P. Dupont, La statuaire préangkorienne, p. 191)

Dans l'ouvrage quiil consacre à la statuaire préangkorienne, Pierre Dupont ${ }^{1}$ évoque à plusieurs reprises l'image du Buddha en ronde bosse qu'il observait encore en place, au Vat Kompong Luong, peu de temps avant son transfert au musée de Phnom Penh en 1944². Il identifie bien une ouvre de la période du Phnom Da (vII" s.), mais toujours son commentaire bute sur les restaurations qui masquaient l'état originel de la statue. L'œuvre avait subi de nombreuses transformations: l'usnissa était augmentée d'un cône, le cou paraissait anormalement long. Les traits du visage sans relief semblaient totalement repris; les mains, entièrement remodelées, étaient très grossières avec des doigts tubulaires et de même dimension pour la main en pronation. C'est en fait tout le modelé qui était affadi par les recouvrements successifs d'enduits et laques. Au moment de son entrée au musée, l'œuvre a encore été recouverte de trois couches de peintures synthétiques (fig. 1).

La statue qui a toujours fait l'objet d'une présentation privilégiée, fut, dans un premier temps, exposée dans un hall ouvrant au sud-est du musée. Elle est restée au même emplacement jusqu'en 1968, date à laquelle furent entrepris d'importants travaux de restructuration du bâtiment. La statue fut alors déplacée une nouvelle fois pour être bien mise en valeur à l'entrée de la galerie sud et n'a plus quitté cet endroit jusqu'à notre intervention.

Au début de l'année 2000, M. Khun Samen, directeur du Musée national de Phnom Penh, invita l'atelier de restauration à examiner l'œuvre et à envisager une " dérestauration ». L'étude de la stratigraphie des couches successives (fig. 2) révéla, sous les surpeints récents, d'épaisses couches d'enduits et laques correspondant à d'importants rajouts et remodelages. Le mortier qui paraissait aussi dur que du ciment s'avéra, à l'analyse, être un mélange traditionnel à base de coquillages concassés, d'amidon de riz et de suc de palme. Plus en-dessous, au contact de la pierre, on décelait plus indistinctement d'anciens enduits noirs et des laques rouges sur une épaisseur beaucoup plus fine (fig. 2: phases I et II).

Dans un premier temps nous avons retiré au scalpel les peintures synthétiques déjà très écaillées (phase IV ). Progressive-
Fig. 1

Buddha de Vat Kompong Luong. photographié peu après son entrée au musée: recouvert d'une peinture dorée. il a les lobes d'oreille et les genoux allongés,

la tête surélevée,

le faciès remodelé. la main droite refaite. et une usnissa surélevée [phase IV]

(phot. ex-Musée Albert Sarraut. vers 1945)
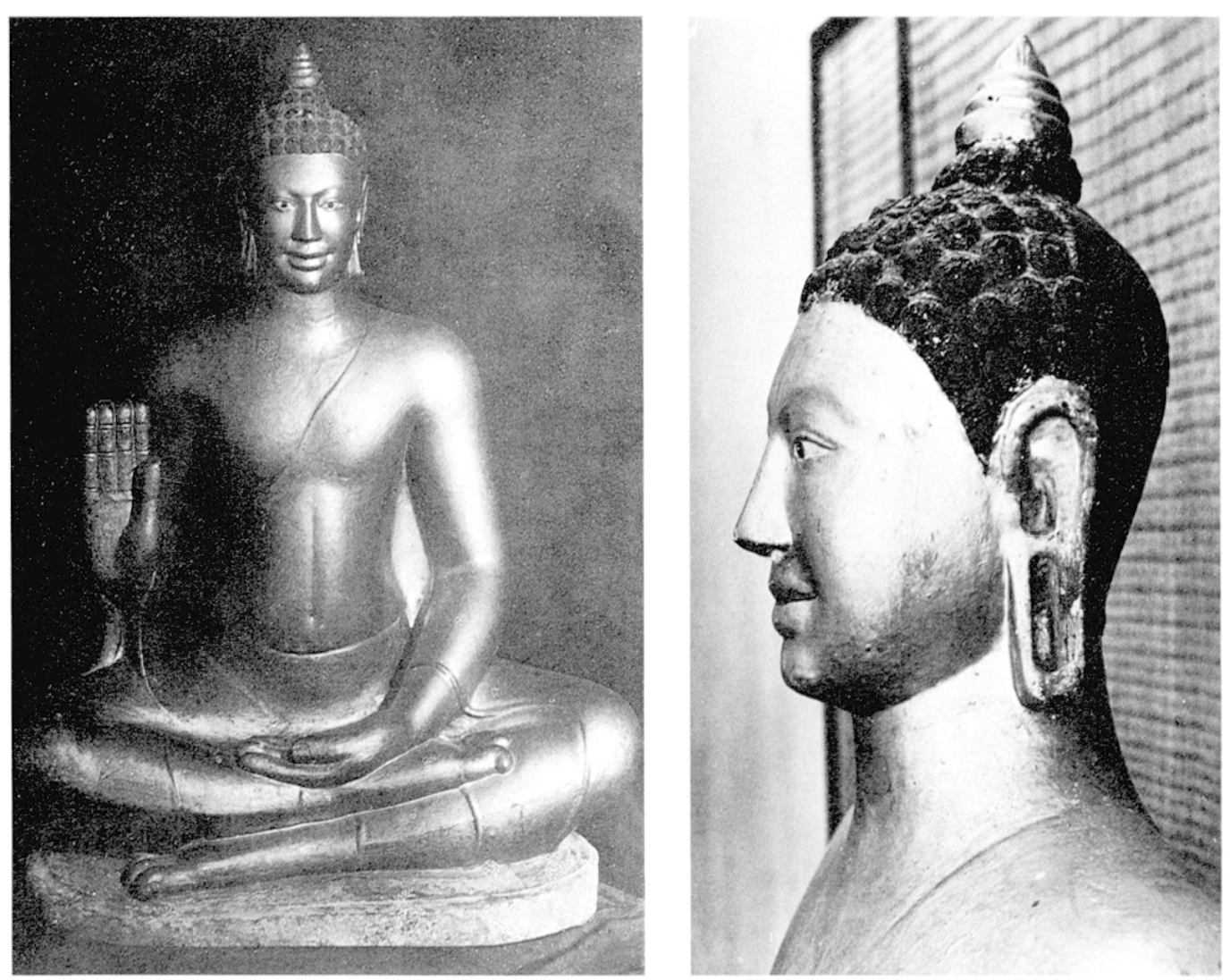


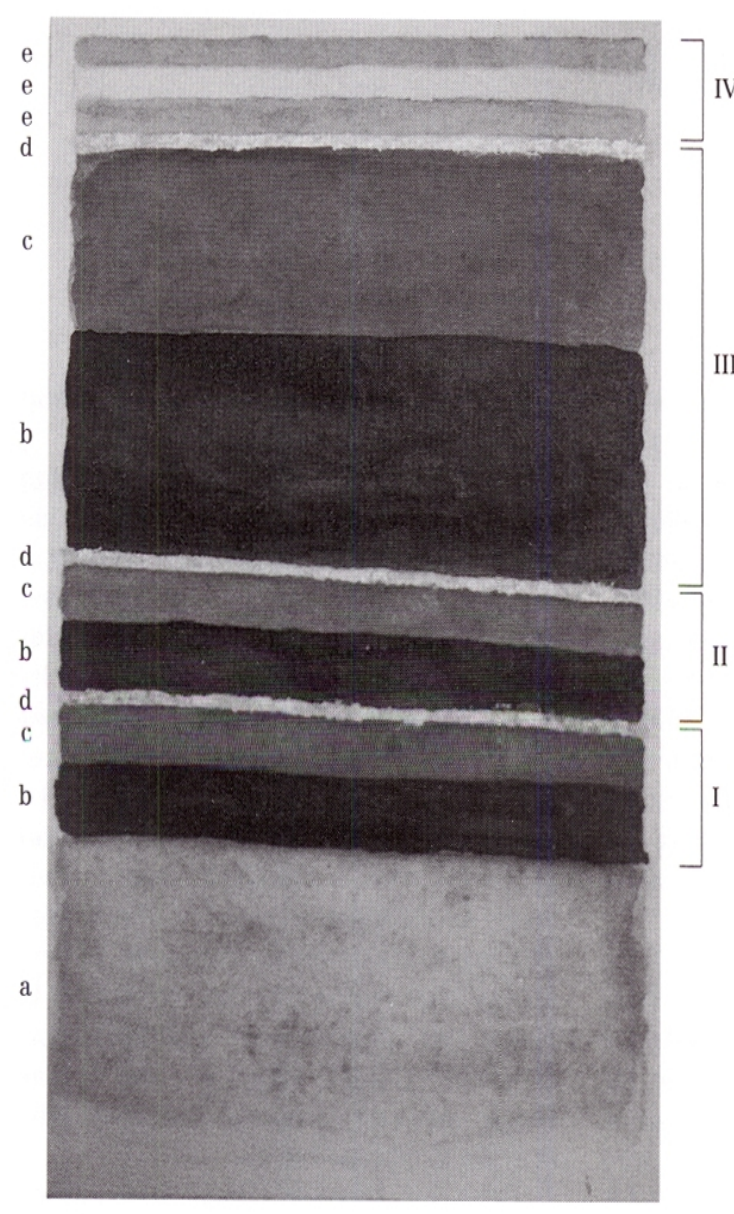

Fig. 2

Diagramme stratigraphique des couches d'enduits et

laque progressivement ajoutées au cours des siècles (schéma atelier de restauration

du Musée national de Phnom Penh)

Les phases I et II sont fines: moins d'1/2 mm.

La phase III est beaucoup plus épaisse: de $3 \mathrm{~mm}$ à plus d'1 cm. Sur les parties remodelées, un mortier s'intercale entre les phases II et III.

a: pierre (grès gris vert)

b: enduit noir

c: laque rouge

d: dorure

e: peinture dorée synthétique

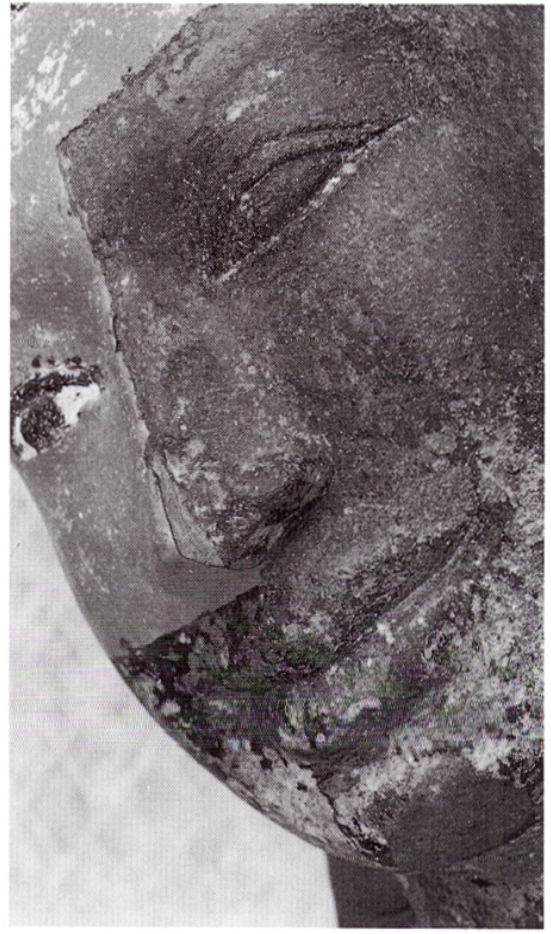

Fig. 4

La tête au cours de la deuxième partie de la restauration. La partie droite du visage porte encore des surmodelages (nez)

et l'épaisse couche d'enduit et de laque [phase III] (phot. atelier de restauration du Musée national de Phnom Penh) ment nous avons mis à jour un état très proche de celui dont témoigneront les vieux achars du Vat Kompong Luong interrogés plus tard. L'image apparaissait essentiellement d'un rouge vif, de la couleur de la laque (fig. 3). Au regard de la facture des remodelages, cet état ${ }^{3}$ serait daté du début du XXe s. Un joint fermé au ciment apparaissait aussi autour de la taille et du bras droit, révélant que la sculpture avait été brisée en deux (couche supérieure de la phase III)

A ce stade, un choix déterminant devait être fait. Des arguments religieux poussaient à conserver l'œuvre dans cet état finalement assez grossier, voire peut-être avec une harmonisation de la dorure. Aller plus loin dans la « dérestauration » risquait

Fig. 3

Le Buddha en 2000, après la première partie de la restauration qui fait apparaître la couche de laque rouge [phase III] se présente à peu près dans l'état où il était en 1944; l'uṣniṣa, le cou surélevé,

les genoux remodelés et les doigts allongés sont encorc cn place (phot. Darren Campbell)

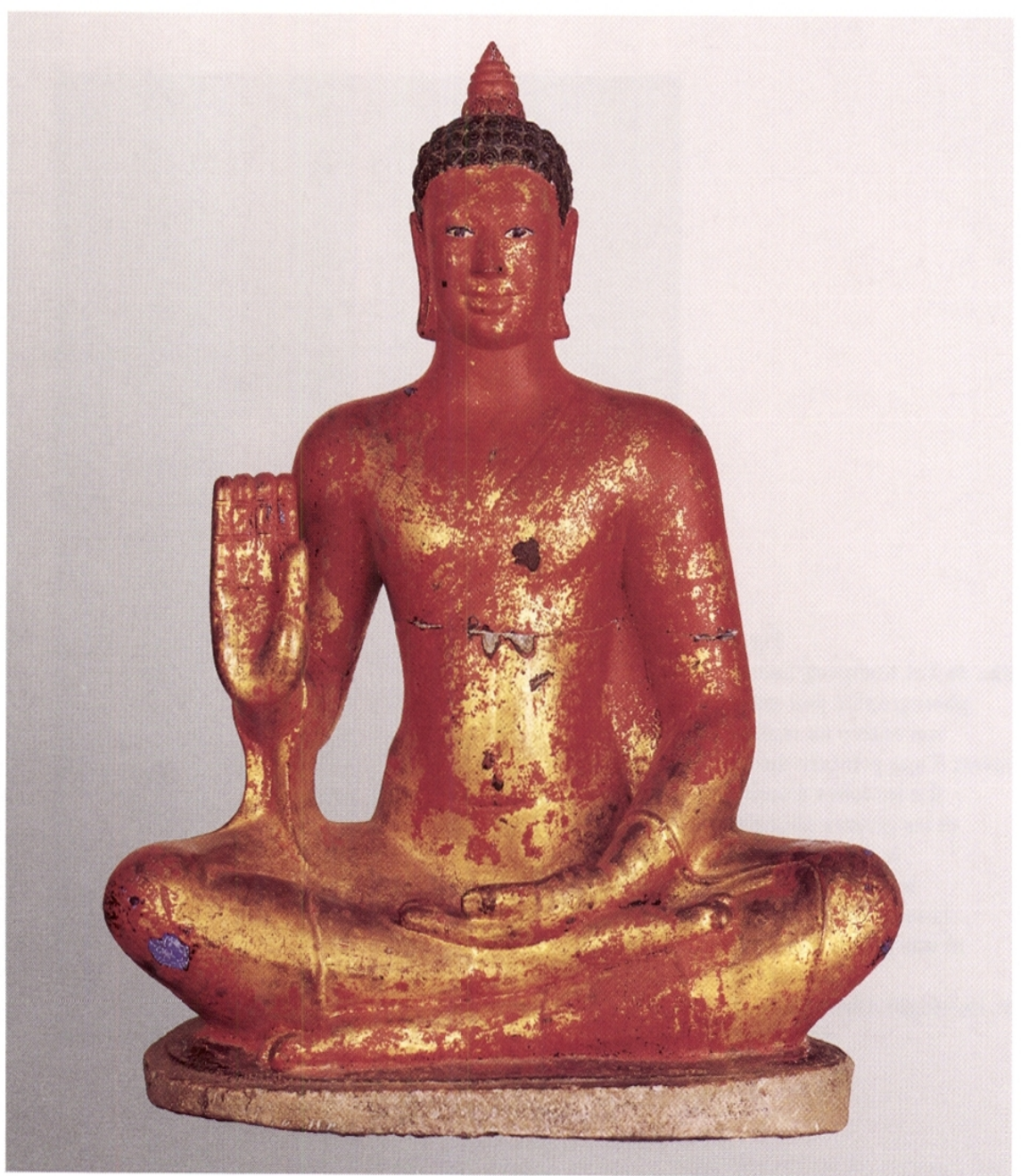




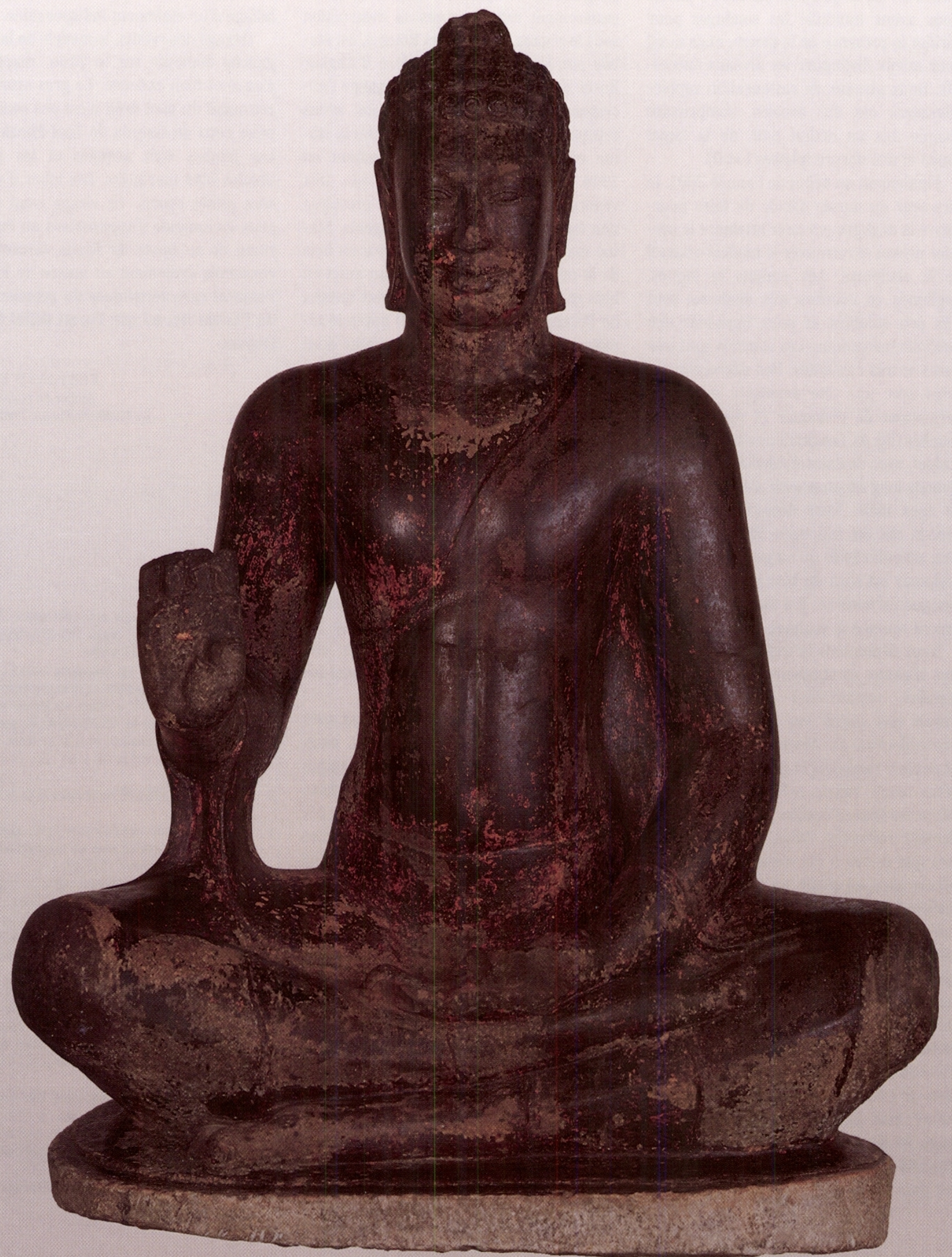

Fig. 5

Le Buddha en 2001, au terme de la restauration,

avec sa forme d'origine et les restes des premiers enduits [phase I]

tel qu'il est actuellement exposé

au Musée de Phnom Penh (phot. Darren Campbell) 
de révéler une image très altérée. Pendant près d'un an la question est restée posée. Nous avons multiplié les sondages pour vérifier la présence de la pierre, mais aussi pour mieux distinguer les niveaux successifs. Deux niveaux de restauration supplémentaires ont été décelés, comprenant chaque fois un enduit noir, de la laque rouge et une dorure (phases I et II).

Finalement au début de l'année 2001, le directeur du musée décide de faire poursuivre le dégagement pour atteindre le premier niveau et retrouver le modelé originel de la sculpture. Les enduits et laques, appliqués en couches très épaisses, sont très peu solubles et nous imposent une méthode mécanique. Les couches sont très dures et très cassantes. Des micro-percussions avec une tête émoussée de ciseau permettent de disloquer et dissocier ces couches (fig. 4). Les finitions s'effectuent au scalpel, avec de l'alcool éthylique. Ce dégagement long et progressif s'étale sur plus de deux mois. Nous découvrons que les genoux ont été rallongés. En fait un examen attentif révèle qu'au genou gauche, le sculpteur est sorti du bloc initial et qu'il a manqué de matière; il a malgré tout habilement réussi à le sculpter.

Nous démontons la cassure de la taille, plus récente, et purgeons les ciments. La dernière restauration importante, qui n'avait pas lésiné sur les interventions (probablement au début du $\mathrm{xx}^{\mathrm{e}}$ s.), avait totalement remodelé le genou gauche et du même coup repris celui de droite. De même, les lobes d'oreilles avaient été sensiblement rallongés. Nous découvrons encore que le cou a été cassé. La tête a été refixée avec un tenon en bois et un joint épais qui la surélevait de près de trois centimètres. Les doigts de la main droite, disparus, ont été remontés sur une âme en cuivre. De même l'usnișa conique a été superposée sur la protubérance crânienne. Enfin l'ancien visage se dévoile, beaucoup mieux conservé que nous aurions pu le craindre. A la surface de la pierre désormais, il reste essentiellement le premier enduit ${ }^{4}$ noir, parsemé de points de laque rouge et de dorure (phase I). La statue apparaît maintenant avec un beau lustre noir dans son modelé d'origine (fig. 5).
Nous nous sommes rendu au Vat Kompong Luong en septembre 2001. La pagode, entièrement rebâtie après sa destruction sous le régime des Khmers Rouges, est établie sur les bords de la rivière d'Angkor Borei, au cœur même de l'enceinte de l'ancienne cité du même nom. Nous avons retrouvé deux achars qui ont connu la statue en place, avant son enlèvement en 1944. L'image était alors, selon eux, très vénérée, et son enlèvement avait provoqué une forte émotion chez les villageois. Statue majeure d'un des deux vihāra en bois de la pagode, elle reposait sur un autel en bois garni de verroterie, entre des images de Preah Kô et Preah Ke0 ${ }^{5}$. D'après le témoignage des deux achars, la statue était encore dorée, laissant apparaître les couches de laque rouge sous-jacente (niveau supérieur de la phase III). La statue n'était pas cassée à la taille. Cette cassure que nous observons aujourd'hui s'est produite lors du transport de la statue vers Phnom Penh. Le plan de cassure est très régulier et correspond à un lit de la roche sans doute déjà très fragilisé. La pièce a donc été remontée pour permettre sa présentation; c'est ce qui explique probablement les peintures appliquées (phase IV) pour dissimuler le joint de cassure; elle apparaît ainsi fraîchement repeinte sur les premières photos (fig 1).

Le Buddha de Kompong Luong est assis à l'indienne (virāsana) faisant de la main droite le geste dit de l'absence de crainte (abhayamudrā $)^{6}$. Il fait corps avec une base d'épaisseur variable et plutôt faible (de 5 à $10 \mathrm{~cm}$ ) de plan ovale. Le dessus de la base présente un léger méplat et son pourtour est juste épannelé. Les enduits n'ayant jamais été appliqués sur les côtés, on peut penser qu'elle était vraisemblablement encastrée. Au-dessous, il n'y a pas de tenon d'ancrage; en effet, la posture qui abaisse le centre de gravité, et l'importante surface d'assise, ne nécessitent pas ce dispositif.

Le Bienheureux a une constitution puissante, marquée par de larges épaules et des membres épais. La samighātị laisse dégagée l'épaule gauche, et son rebord, qui passe sur la poitrine, est sinueux. L'antaravāsaka est marqué à la ceinture et aux chevilles. Un drapé passe autour de l'avant-bras droit: il semble qu'il n'ait pas de fondement véritable et constitue l'habillage d'un étaiement indispensable.

Dégagé des refaits, le modelé de la main gauche déployée sur le giron, réapparaît élancé et bien cadencé. Le gros orteil très retroussé du pied droit n'est pas sans rappeler ceux du Ganeśa de Tuol Pheak Kin ${ }^{i}$. Les jambes sont arquées et les arêtes tibiales bien marquées. Les lobes d'oreille sont plutôt courts. Le visage rond et les yeux en amande s'apparentent au Paraśurāma et au buste de Kṛșna Govardhana conservés également au musée de Phnom Penh $^{8}$ et caractéristiques du premier style du Phnom Da, tel que l'avait défini Pierre Dupont.

BERTRAND PORTE: Atelier de restauration du Musée de Phnom Penh/EFE0

Notes

1 P. Dupont, La statuaire préangkorienne. Ascona, Artibus Asiae, 1955; pages 189-210: les statues préangkoriennes du Buddha.

2 En provenance du Vat Kompong Luong (Angkor Borei, Takeo), ce Buddha en grès gris vert à grain assez fin date du vil" s. Entré au Musée national de Phnom Penh le 25.09.1944, il porte les $\mathrm{n}^{\circ}$ B 701 (cote Groslier) et B10.31 (cote Boisselier), et mesure $105 \times 97 \times 53 \mathrm{~cm} . \mathrm{N}^{\circ}$ d'inventaire: Ka 1731.

3 Un témoin est aujourd'hui conservé au revers de la statue

4 Une analyse par radiocarbone (réf.: Lyon- 1718 [OX $]$ ]) date cet enduit avec un maximum de probabilités entre 1415 et 1515 ap. J.-C.

5 "Le saint taureau et le saint cristal » : représentations d'un mythe toujours bien vivant, réunissant l'image du Buddha et celle du taureau. $C f$. ANG Choulean, "Nandin et ses avatars ", in Ang. kor et dix siècles d'art khmer, Paris, Réunion des Musées Nationaux, 1997, p. 63-69.

6 Cf. le Buddha de Mankuvar, voir James C. Harle, Gupta Sculpture. Indian Sculpture of the Fourth to the Sixth Centuries A.D., London, Oxford University Press, 1974 [reprint: New Delhi, Munshiram Manoharlal, 1996], pl. 55 et p. 45 (information du Pr B. Dagens)

7 Musée de Phnom Penh, Ka 1588. Voir Angkor et dix siècles d'art khmer, op.cit., p. 181, et Les Ganesa du Musée national de Phnom Penh. Phnom Penh, éditions du Mékong et Musée national de Phnom Penh, 2000, p. 11

8 Ka 1608 et Ka 1641, dernièrement restaurés. 